\title{
Antiepileptic Drug Titration and Related Health Care Resource Use and Costs
}

\author{
Jesse Fishman, PharmD; Linda Kalilani, PhD; Yan Song, PhD; \\ Elyse Swallow, MPP; and Imane Wild, PhD
}

\begin{abstract}
BACKGROUND: Unexpected breakthrough seizures resulting from suboptimal antiepileptic drug (AED) dosing during the titration period, as well as adverse events resulting from rapid $A E D$ titration, may influence the titration schedule and significantly increase health care resource use (HRU) and health care costs.

OBJECTIVE: To assess the relationship between AEDs, HRU, and costs during AED titration and maintenance.

METHODS: Practicing neurologists were recruited from a nationwide panel to provide up to 3 patient records each for this retrospective medical chart review. Patients with epilepsy who were aged $\geq 18$ years and had initiated an AED between January 1, 2014, and January 1, 2016, were followed for 6 months from AED initiation. Titration duration was the time from AED initiation to the beginning of treatment maintenance as determined by the physician. Outcomes were epilepsy-specific HRU (hospitalizations, emergency department visits, outpatient visits, physician referral, laboratory testing/diagnostic imaging, and phone calls) and related costs that occurred during the titration or maintenance treatment periods.
\end{abstract}

RESULTS: Of 811 patients, $156,128,125,120,114,107$, and 61 initiated the following AEDs: levetiracetam, lamotrigine, lacosamide, valproate, topiramate, carbamazepine, and phenytoin, respectively. Most patients (619/803 [77.1\%] with complete AED data) received monotherapy. Baseline characteristics were similar across AEDs (mean [SD] age, 36.6 [14.4] years; $59.0 \%$ male). Kaplan-Meier estimates of titration duration ranged from 3.3 weeks (phenytoin) to 8.1 weeks (lamotrigine). From titration to maintenance, the overall incidence of HRU per person-month decreased $54.5 \%$ 89.3\% for each HRU measure except outpatient visits (24.6\% decrease). Total epilepsy-related costs decreased from $\$ 80.48$ to $\$ 42.77$ per personmonth, or $46.9 \%$ from titration to maintenance.

CONCLUSIONS: AED titration periods had higher HRU rates and costs than AED maintenance, suggesting that use of AEDs with shorter titration requirements reduces health care costs, although disease severity may also factor into overall cost.

J Manag Care Spec Pharm. 2018;24(9):929-38

Copyright $\odot 2018$, Academy of Managed Care Pharmacy. All rights reserved.

\section{What is already known about this subject}

Titration of an antiepileptic drug (AED) is a critical period during which the desire to quickly attain an effective dose must be balanced with the need to minimize side effects, which typically dictates a slower titration.

During titration, subtherapeutic drug levels (e.g., because of lower dosing or from poor adherence due to side effects) may result in seizures, with consequences for patients and health care resource use (HRU) and costs.

\section{What this study adds}

In this cohort of patients with epilepsy, the AED titration period was associated with a $>4$-fold higher risk of any seizure and higher HRU rates and costs, compared with the maintenance period. AED titration involves high HRU; during maintenance, most HRU items decreased by $50 \%-90 \%$ and associated costs decreased by nearly $50 \%$ compared with titration.

Confirmation is needed to ascertain whether AEDs without a titration requirement provide patient-centered benefits while minimizing costs.

$\square_{i}$ pilepsy, a collection of neurological disorders characterized by seizures, affects approximately 3.4 million people in the United States and 50 million people around the world. ${ }^{1,2}$ Epilepsy carries a 2- to 11-fold increased risk of premature death compared with the general population, ${ }^{3,4}$ with injury, sudden unexpected death in epilepsy, and status epilepticus contributing to the increased risk. ${ }^{4}$ Epileptic seizures are classified as generalized seizures, which occur in both brain hemispheres; focal seizures, which originate in a specific brain area and can spread to both hemispheres (resulting in secondary generalization); or unknown onset seizures. ${ }^{5}$ Different epilepsy types require different treatment modalities to reduce seizure recurrence and improve patient quality of life. ${ }^{6}$ The most common treatment is with antiepileptic drugs (AEDs), although surgery may be an option for drug-resistant epilepsy.

Patients newly diagnosed with epilepsy are typically treated with AED monotherapy. ${ }^{7-9}$ If AED monotherapy fails to produce freedom from seizures, patients are switched to another AED monotherapy or receive adjunctive therapies. ${ }^{10,11}$ Because the response to AEDs differs for individual patients, most AEDs are titrated to an optimal dosage to minimize adverse events 
and improve tolerability. Titration schedules vary for each AED and depend on several factors, including whether the AED is administered as monotherapy or adjunctive therapy, the pharmacokinetic profile of the AED, the patient's response to treatment, the adverse event profile of the AED, and potential drug-drug interactions.

Because of this variability in titration across AEDs, a health care provider's titration strategy often combines a patient's characteristics, individual experience with a given AED, and the titration schedule specified in the drug labels. ${ }^{12}$ Assessment of serum drug concentration (necessary for some AEDs to monitor the dose) as well as disease severity, seizure frequency, and side effects affect treatment success, particularly given the wide range of AED titration periods. ${ }^{13,14}$

For many AEDs, U.S. Food and Drug Administration labeling recommends fairly slow titration (2-6 weeks on average), laboratory testing, and therapeutic drug monitoring. However, these recommendations are based on regulatory trials in which drugs are started rapidly; in clinical practice, it has been recommended to slow down titration up to 2-fold. ${ }^{15-17}$

The duration of AED titration periods may be associated with underrecognized economic consequences. Titration can potentially lead to lower adherence and higher health care resource use (HRU) and health care costs. ${ }^{18}$ Suboptimal AED dosing during titration can lead to breakthrough seizures, ${ }^{19}$ and unexpected breakthrough seizures associated with lack of AED efficacy can significantly increase HRU and costs. ${ }^{20}$ In some cases, rapid titration can lead to the occurrence or exacerbation of adverse events and thereby affect treatment adherence. ${ }^{12}$ Given the tradeoff between efficacy and tolerability during the titration phase of a newly initiated AED, lack of efficacy and/ or adverse events may influence the titration schedule and thus affect HRU and costs.

To our knowledge, no studies using real-world data have investigated the relationship between AED titration and HRU and costs. Understanding the effect of AED titration will be important for assessing the value of novel AEDs that do not require dosing titration. ${ }^{21}$ This study sought to describe the time from AED initiation to reaching a physician-assessed maintenance dose (duration of titration) for specific AEDs, and to describe clinical outcomes, HRU, and health care costs associated with dose titration during the 6-month period following AED initiation.

\section{Methods}

\section{Study Design}

This retrospective cohort study collected data from a medical record review. General neurologists practicing in the United States were recruited from an established panel of verified physicians, maintained by Schlesinger Associates (Iselin, NJ), who agreed to receive invitations to participate in online data collection. Participating physicians are routinely monitored for data completeness and quality across multiple studies, with underperformance on quality metrics leading to removal from the panel. Physicians were eligible for study participation if they had treated at least 1 patient with epilepsy who satisfied the eligibility criteria detailed below.

To reduce potential selection bias, each physician was instructed to randomly select up to 3 eligible patients. Participating physicians collected patient information using an electronic case report form through a secure online portal. To collect a sample balanced among commonly prescribed AEDs (i.e., carbamazepine, lamotrigine, levetiracetam, valproate/valproic acid, topiramate, lacosamide, and phenytoin), data collection was staged such that the first quarter of cases included any of these AEDs. Overrepresented AEDs were removed from the inclusion criteria list for subsequent rounds of data collection.

Because this study was a retrospective chart review from de-identified patients, an exemption from a full institutional review board review was granted by the New England Institutional Review Board. The researchers were blinded to the identity of the participating physicians and patients; likewise, physicians were blinded to the identity of the researchers.

\section{Study Population}

Patients were eligible for inclusion if they had a diagnosis of epilepsy as confirmed by the physician and had initiated a commonly prescribed AED (listed above) as either monotherapy or adjunctive therapy between January 1, 2014, and January 1, 2016. The first AED initiated within this period was defined as the index AED; the date of index AED initiation was defined as the index date. Patients were required to be aged $\geq 18$ years on the AED index date and not have been titrated on another AED for 1 month on or before the AED index date. Physicians were required to have access to their patients' epilepsy-related medical records for at least 6 months before and after the index date. Information on index AED dose titration, number of seizures between clinic visits, and epilepsy-related hospitalization and emergency room visits were required to be assessable through the medical records. Medical record abstraction was completed in October 2016.

Patient characteristics included demographics (age, sex, race, and insurance type), neuropsychiatric and other chronic comorbidities, characteristics of epilepsy (time since first epilepsy diagnosis, seizure type and frequency, and status epilepticus history), and epilepsy treatment history before index date (prior AED use, concomitant AED use, vagal nerve stimulation, and epileptic surgery). Charlson Comorbidity Index (CCI) was derived based on the comorbidity information collected.22 


\section{Study Outcomes}

Study outcomes included treatment patterns of the index AED, seizure frequency, and epilepsy-related HRU and health care costs. Treatment patterns of the index AED included starting dose, physician-assessed maintenance dose, and time from index AED initiation to physician-assessed maintenance dose (i.e., titration duration), which was reported by physicians based on information recorded in patient records.

Seizures by specific types (focal aware [simple partial], focal impaired awareness [complex partial], focal to bilateral tonicclonic [secondary generalized], and generalized) ${ }^{5,23}$; epilepsyrelated HRU (hospitalization, emergency department visits, outpatient visit, laboratory orders, and phone calls to office personnel); and associated health care costs were evaluated during titration and maintenance period within 6 months following the index date. Emergency department visits that resulted in hospitalization were counted as both an emergency department visit and a hospitalization. Health care costs were estimated based on the incidence rates and unit costs of HRU derived from a previous study. ${ }^{24}$ All costs were adjusted to 2016 U.S. dollars using the medical care component of the Consumer Price Index.

The unit cost per epilepsy-related hospitalization, emergency department visit, outpatient visit, and order for laboratory tests/diagnostic imaging was $\$ 705.77, \$ 315.26$, $\$ 128.77$, and $\$ 60.31$, respectively. ${ }^{24}$ Unit cost per order for laboratory test or diagnostic imaging was calculated as the weighted average of the unit costs of laboratory tests (AED blood levels and other laboratory tests) and diagnostic procedures (electroencephalogram, computed tomography scan, magnetic resonance imaging, other radiologic procedures, lumbar puncture, and neuropsychological testing). The unit costs per referral and phone call to office personnel were assumed to be zero.

\section{Sample Size}

During the study design, a sample size of 800 was calculated to provide sufficient power to detect differences in mean number of physician office visits (a key HRU of interest) per patient over the 6-month period after treatment initiation for 3 commonly used AEDs (lamotrigine, levetiracetam, and carbamazepine, which represent AEDs with different mechanisms of action and approval times). The power calculation assumed a Poisson distribution with independent samples for the total number of visits.

\section{Statistical Analysis}

Patient demographics, comorbidities, disease history, epilepsy characteristics, and epilepsy treatment history were described in the overall sample and by index AED. Means, standard deviations (SDs), medians, and interquartile ranges were calculated for continuous variables. Frequencies and percentages were calculated for categorical variables. Time from AED index date to physician-assessed maintenance dose was estimated using the Kaplan-Meier method. Patients who did not reach physician-assessed maintenance dose were censored at the end of their follow-up. Patients who discontinued index AED before reaching maintenance dose were considered to have not reached maintenance dose.

The incidence rates of seizures and HRU (number of events per person-month) during the titration or maintenance period were calculated as the sum of events over the sum of person-months during titration or maintenance period within the 6 months following the index date. ${ }^{25}$ Epilepsy-related health care costs per person-month during the titration or maintenance period were calculated as the product of the unit cost of each type of HRU and the incidence rates assessed during the titration or maintenance period.

Comparison of the incidence rates of seizures and epilepsyrelated HRU, and health care costs per person-month during titration versus maintenance were modeled using adjusted generalized linear regression models that account for correlations between outcomes during the titration and maintenance periods collected from the same patient. Separate models were fit for each outcome. Log link functions and negative binomial (for incidence rates) or Tweedie distributions (for costs) were specified in the regression models. ${ }^{26}$

Covariates included index AED, age on index date, sex, time from first diagnosis of epilepsy to index date, CCI, history of status epilepticus, prior vagal nerve stimulation, prior epileptic surgery, total number of seizures during baseline, any concomitant AED use on index date, any HRU during baseline, and number of outcome events that occurred during baseline (e.g., number of hospitalizations during baseline was adjusted for when incidence of hospitalization was modeled as the outcome). Patients with any missing values in the covariates were excluded from that analysis. Because missing covariate values occurred in $<10 \%$ of the population, excluding these patients is not expected to bias the analyses. Adjusted incidence rate ratios and 95\% confidence intervals (CIs) were reported for seizure outcomes and epilepsy-related HRUs. Cost ratios and 95\% CIs were reported for health care costs.

Statistical analyses were performed using SAS software package 9.4 (SAS Institute, Cary, NC).

\section{Results}

\section{Patient Characteristics}

A total of 811 eligible patients with epilepsy were included in the analysis, consisting of those initiating levetiracetam $(n=156)$, lamotrigine $(n=128)$, lacosamide $(n=125)$, valproate $(n=120)$, topiramate $(n=114)$, carbamazepine $(n=107)$, and phenytoin $(n=61)$. Patient characteristics were generally consistent across index AED groups (Table 1); exceptions included a slightly older age for phenytoin, the proportion of males was lowest for topiramate and highest for valproate, and lack of insurance was higher in the valproate and phenytoin groups compared with the other index AED groups. 
Antiepileptic Drug Titration and Related Health Care Resource Use and Costs

TABLE 1 Patient Characteristics for the Overall Population and Individual AED Groups

\begin{tabular}{|c|c|c|c|c|c|c|c|c|}
\hline & Overall & Levetiracetam & Lamotrigine & Lacosamide & Valproate & Topiramate & Carbamazepine & Phenytoin \\
\hline Baseline Characteristic & $N=811$ & $\mathrm{n}=156$ & $\mathrm{n}=128$ & $\mathrm{n}=125$ & $\mathrm{n}=120$ & $\mathrm{n}=114$ & $\mathrm{n}=107$ & $\mathrm{n}=61$ \\
\hline Age, years, mean $\pm S D$ & $36.6 \pm 14.4$ & $38.5 \pm 14.6$ & $35.4 \pm 14.1$ & $36.2 \pm 14.5$ & $34.4 \pm 14.8$ & $36.0 \pm 12.4$ & $35.6 \pm 14.5$ & $42.3 \pm 15.2$ \\
\hline Male, ${ }^{a} \mathrm{n}(\%)$ & $477(59.0)$ & $104(67.5)$ & $61 \quad(47.7)$ & $70(56.0)$ & $89(74.2)$ & $47 \quad(41.6)$ & $65(60.7)$ & $41 \quad(67.2)$ \\
\hline $\begin{array}{l}\text { Charlson Comorbidity } \\
\text { Index, mean } \pm \text { SD }\end{array}$ & $0.4 \pm 1.1$ & $0.4 \pm 1.0$ & $0.3 \pm 0.7$ & $0.6 \pm 1.3$ & $0.3 \pm 0.9$ & $0.4 \pm 1.1$ & $0.3 \pm 0.9$ & $0.7 \pm 1.6$ \\
\hline \multicolumn{9}{|l|}{ Race, ${ }^{\text {a }}$ n (\%) } \\
\hline White & $604(75.0)$ & $129(84.3)$ & $97(76.4)$ & $89(71.2)$ & $91 \quad(75.8)$ & $80(70.8)$ & $79(73.8)$ & $39(65.0)$ \\
\hline Black & $119(14.8)$ & $15 \quad(9.8)$ & $20(15.7)$ & $18(14.4)$ & $17(14.2)$ & $16(14.2)$ & $18(16.8)$ & $15(25.0)$ \\
\hline Asian & $35 \quad(4.3)$ & $6 \quad(3.9)$ & $6 \quad(4.7)$ & $9 \quad(7.2)$ & $3 \quad(2.5)$ & $5 \quad(4.4)$ & $4 \quad(3.7)$ & $2 \quad(3.3)$ \\
\hline Hispanic or Latino & $43 \quad(5.3)$ & $2 \quad(1.3)$ & $3 \quad(2.4)$ & $8 \quad(6.4)$ & $9 \quad(7.5)$ & $11 \quad(9.7)$ & $6 \quad(5.6)$ & $4 \quad(6.7)$ \\
\hline Other & $4 \quad(0.5)$ & $1 \quad(0.7)$ & $1 \quad(0.8)$ & $1 \quad(0.8)$ & $0 \quad(0.0)$ & $1 \quad(0.9)$ & $0 \quad(0.0)$ & $0 \quad(0.0)$ \\
\hline \multicolumn{9}{|l|}{ Insurance type, ${ }^{b}$ n (\%) } \\
\hline $\begin{array}{l}\text { Commercial/private } \\
\text { insurance }\end{array}$ & $510(65.0)$ & $108(75.0)$ & $88(68.8)$ & $75(62.5)$ & $66(55.9)$ & $76(68.5)$ & $65 \quad(61.9)$ & $32(54.2)$ \\
\hline Medicare & $114(14.5)$ & $20(13.9)$ & $16(12.5)$ & $24(20.0)$ & $15(12.7)$ & $13(11.7)$ & $16(15.2)$ & $10 \quad(16.9)$ \\
\hline Medicaid & $149(19.0)$ & $16(11.1)$ & $23(18.0)$ & $24(20.0)$ & $32(27.1)$ & $18(16.2)$ & $22(21.0)$ & $14(23.7)$ \\
\hline $\begin{array}{l}\text { Military insurance } \\
\text { (VA or active military) }\end{array}$ & $16 \quad(2.0)$ & $2 \quad(1.4)$ & $5 \quad(3.9)$ & $1 \quad(0.8)$ & $2 \quad(1.7)$ & $5 \quad(4.5)$ & $1 \quad(1.0)$ & $\begin{array}{ll}0 & (0.0)\end{array}$ \\
\hline Other & $3 \quad(0.4)$ & $0 \quad(0.0)$ & $0 \quad(0.0)$ & $0 \quad(0.0)$ & $1 \quad(0.8)$ & $1 \quad(0.9)$ & $1 \quad(1.0)$ & $0 \quad(0.0)$ \\
\hline No insurance & $19 \quad(2.4)$ & $1 \quad(0.7)$ & $1 \quad(0.8)$ & $2 \quad(1.7)$ & $6 \quad(5.1)$ & $3 \quad(2.7)$ & $3 \quad(2.9)$ & $3 \quad(5.1)$ \\
\hline \multicolumn{9}{|l|}{ Epilepsy history } \\
\hline $\begin{array}{l}\text { Time since first diagno- } \\
\text { sis of epilepsy to index } \\
\text { date, years, median (1st } \\
\text { quartile-3rd quartile) }\end{array}$ & $\begin{array}{c}0.80 \\
(0.0-8.0)\end{array}$ & $\begin{array}{c}0.04 \\
(0.0-2.9)\end{array}$ & $\begin{array}{c}1.90 \\
(0.1-9.8)\end{array}$ & $\begin{array}{c}3.90 \\
(0.1-11.3)\end{array}$ & $\begin{array}{c}0.30 \\
(0.0-8.1)\end{array}$ & $\begin{array}{c}1.00 \\
(0.0-8.4)\end{array}$ & $\begin{array}{c}0.30 \\
(0.0-6.0)\end{array}$ & $\begin{array}{c}0.06 \\
(0.0-6.6)\end{array}$ \\
\hline $\begin{array}{l}\text { Number of any seizures } \\
\text { during } 1 \text { month before } \\
\text { index date, }{ }^{\mathrm{a}} \text { median (lst } \\
\text { quartile-3rd quartile) }\end{array}$ & $\begin{array}{c}2.0 \\
(1.0-3.0)\end{array}$ & $\begin{array}{c}2.0 \\
(1.0-3.0)\end{array}$ & $\begin{array}{c}2.0 \\
(1.0-3.0)\end{array}$ & $\begin{array}{c}2.0 \\
(1.0-3.0)\end{array}$ & $\begin{array}{c}2.0 \\
(1.0-3.0)\end{array}$ & $\begin{array}{c}2.0 \\
(1.0-3.0)\end{array}$ & $\begin{array}{c}2.0 \\
(1.0-4.0)\end{array}$ & $\begin{array}{c}2.0 \\
(1.0-3.0)\end{array}$ \\
\hline $\begin{array}{l}\text { History of status } \\
\text { epilepticus, }{ }^{a} \text { n (\%) }\end{array}$ & $79 \quad(9.9)$ & $7 \quad(4.5)$ & $7 \quad(5.5)$ & $15(12.2)$ & $13(10.8)$ & $13(11.7)$ & $11(10.9)$ & $13(21.3)$ \\
\hline \multicolumn{9}{|l|}{ Epilepsy treatment history } \\
\hline $\begin{array}{l}\text { Used AED at any time } \\
\text { before index date, }{ }^{a} \mathrm{n}(\%)\end{array}$ & $408(57.8)$ & $58(42.3)$ & $70(60.3)$ & $90(76.9)$ & $52(52.0)$ & $69(68.3)$ & $45(52.9)$ & $24(48.0)$ \\
\hline \multicolumn{9}{|c|}{ Number of AEDs before index date, ${ }^{\text {a }} \mathrm{n}(\%)$} \\
\hline 0 & $298(42.2)$ & $79 \quad(57.7)$ & $46(39.7)$ & $27(23.1)$ & $48(48.0)$ & $32(31.7)$ & $40 \quad(47.1)$ & $26(52.0)$ \\
\hline 1 & $233(33.0)$ & $40 \quad(29.2)$ & $43(37.1)$ & $39(33.3)$ & $34(34.0)$ & $35(34.7)$ & $28(32.9)$ & $14(28.0)$ \\
\hline 2 & $96(13.6)$ & $14(10.2)$ & $16(13.8)$ & $24(20.5)$ & $10(10.0)$ & $14(13.9)$ & $13(15.3)$ & $5(10.0)$ \\
\hline$\geq 3$ & $79 \quad(9.7)$ & $4 \quad(2.6)$ & $11 \quad(8.6)$ & $27(21.6)$ & $8 \quad(6.7)$ & $20 \quad(17.5)$ & $4 \quad(3.7)$ & $5 \quad(8.2)$ \\
\hline $\begin{array}{l}\text { Used concomitant AED } \\
\text { on the index date, }{ }^{a} \mathrm{n}(\%)\end{array}$ & $184(22.9)$ & $13 \quad(8.4)$ & $39(30.5)$ & $55(44.0)$ & $12(10.2)$ & $36(31.9)$ & $19(18.3)$ & $10(16.7)$ \\
\hline \multicolumn{9}{|c|}{ Number of concomitant AEDs, ${ }^{a}$ n (\%) } \\
\hline 0 & $619(77.1)$ & $142(91.6)$ & $89(69.5)$ & $70(56.0)$ & $106(89.8)$ & $77(68.1)$ & $85(81.7)$ & $50 \quad(83.3)$ \\
\hline 1 & $147(18.3)$ & $11 \quad(7.1)$ & $35(27.3)$ & $39(31.2)$ & $9 \quad(7.6)$ & $28(24.8)$ & $16(15.4)$ & $9(15.0)$ \\
\hline 2 & $26 \quad(3.2)$ & $2 \quad(1.3)$ & $4 \quad(3.1)$ & $12 \quad(9.6)$ & $1 \quad(0.8)$ & $5 \quad(4.4)$ & $2 \quad(1.9)$ & $0 \quad(0.0)$ \\
\hline$\geq 3$ & $11 \quad(1.4)$ & $0 \quad(0.0)$ & $0 \quad(0.0)$ & $4 \quad(3.2)$ & $2 \quad(1.7)$ & $3 \quad(2.6)$ & $1 \quad(1.0)$ & $1 \quad(1.7)$ \\
\hline \multicolumn{9}{|c|}{$\begin{array}{l}{ }^{a} \text { Numbers (\%) were calculated among the patients with nonmissing information. } \\
{ }^{b} \text { More than } 1 \text { insurance type could be selected by the physicians. } \\
A E D=\text { antiepileptic drug; } S D=\text { standard deviation. }\end{array}$} \\
\hline
\end{tabular}

During baseline, a higher proportion of the phenytoin group had a history of status epilepticus compared with other AED groups. Of 706 patients with complete prior AED use data, 408 (57.8\%) had used $\geq 1$ AED at any time before the initiation of the index AED; the levetiracetam and phenytoin groups had the lowest proportion of prior AED use (58/137, 42.3\% and 24/50, $48.0 \%$, respectively). Of 803 patients with complete concomitant AED data, 184 (22.9\%) patients were using $\geq 1$ concomitant AED on the index date and initiated index AED as adjunct therapy; 619 (77.1\%) initiated index AED as monotherapy (Table 1). 


\section{FIGURE 1 Time to Physician-Assessed Maintenance Dose of AEDa}

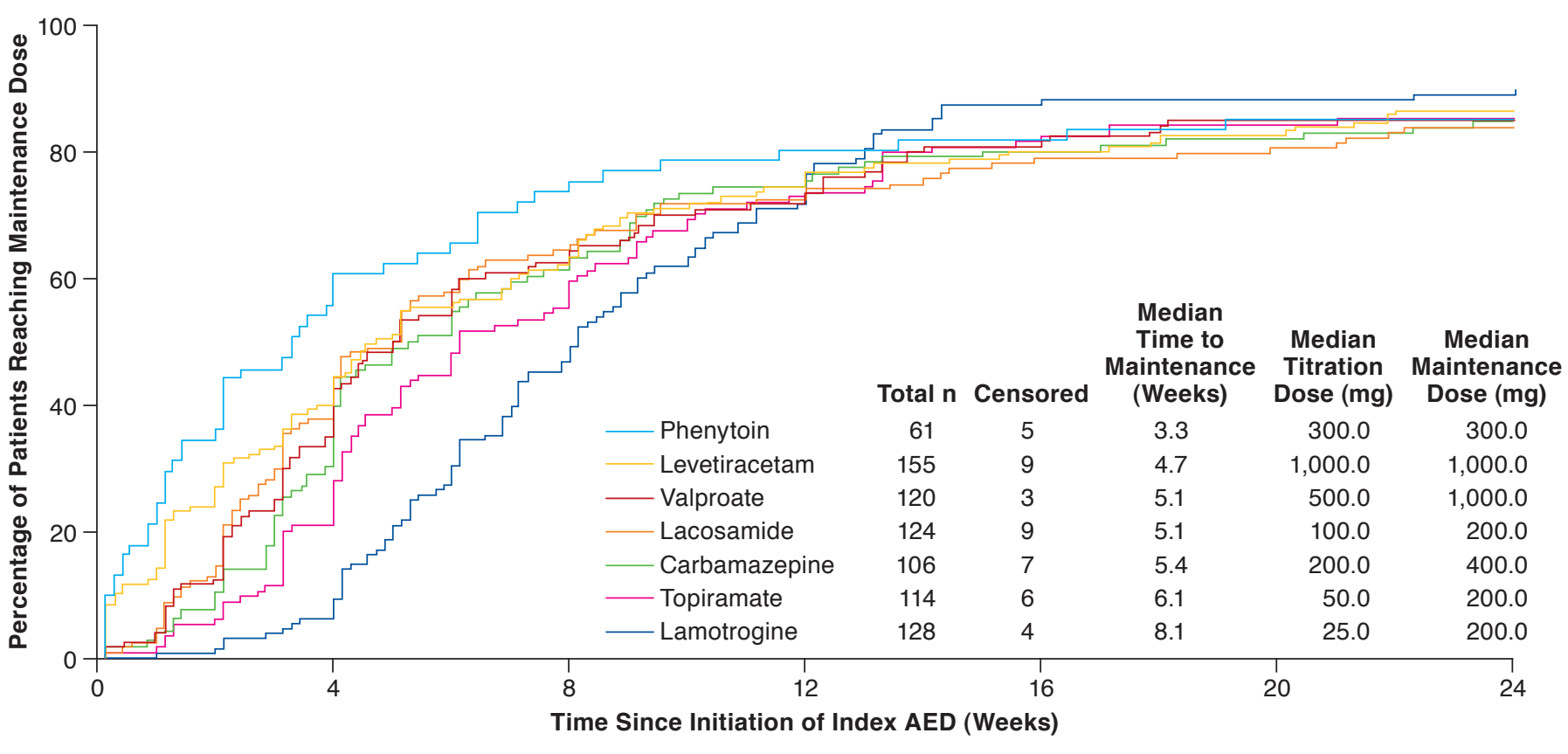

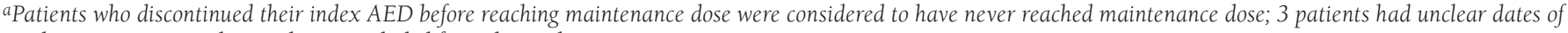
reaching maintenance dose and were excluded from the analysis.

$A E D=$ antiepileptic drug.

Median time from first diagnosis to index AED initiation was the longest for lacosamide (3.9 years) and lamotrigine (1.9 years), and shortest for levetiracetam (0.04 years; 16 days), phenytoin ( 0.06 years; 22 days), and carbamazepine and valproate ( 0.3 years for each; 17 and 14 weeks, respectively; Table 1 ), which may reflect sequential trials of different types of AEDs before index AED, limitations accessing AEDs, or preference based on prior physician experience, titration schedules, and patient needs.

\section{Treatment Patterns of Index AED}

The majority of patients (768/811, 94.7\%; ranging from $91.8 \%$ for phenytoin to $97.5 \%$ for valproate) in the study achieved maintenance dose. Kaplan-Meier analysis demonstrated that the shortest and longest, respectively, median time to maintenance dose was observed for the phenytoin index group (3.3 weeks) and lamotrigine index group (8.1 weeks; Figure 1). Mean (SD) duration of maintenance during 6 months following the index date was 19.9 (5.3) weeks in overall patients, ranging from 18.1 (4.0) weeks for lamotrigine to 21.9 (5.0) weeks for phenytoin.

\section{Clinical Outcomes, HRU, and Health Care Costs}

Of 800 patients with complete seizure data, 212 (26.5\%) experienced seizures during the 6 months following the index date.
The incidence rate of any seizures decreased by $70.3 \%$ from the titration period (32 seizures per 100 person-months) to the maintenance period ( 9.5 seizures per 100 person-months; Table 2), and the incidence rates decreased across all seizure types. After adjusting for covariates, there were significantly higher incidence rates (3- to 5-fold increases) of seizures of all types during the titration versus the maintenance period (Figure 2). Seventy-five patients were excluded from the seizure outcome models due to missing covariates.

Compared with titration, maintenance was associated with lower incidence rates of each type of HRU, with decreases of $89.3 \%, 87.0 \%, 66.2 \%, 54.7 \%, 54.5 \%$, and $24.6 \%$ for laboratory testing/imaging, phone calls, referrals, emergency department visits, hospitalizations, and outpatient visits, respectively (Table 2). Similar results were observed for individual AED groups, with few exceptions. After adjusting for covariates, there were significantly higher rates of all types of HRU during the titration versus the maintenance period (Figure 2).

During titration, the highest HRU costs per person-month were outpatient visits (carbamazepine, \$56.82), laboratory testing/imaging (phenytoin, \$32.27), hospitalizations (carbamazepine, \$31.78), and emergency department visits (phenytoin, \$26.99; Appendix, available in online article). Furthermore, total costs increased by $5 \%$ for each additional month of 
TABLE 2 Incidence Rates of Seizures and Epilepsy-Related Health Care Resource Use During Titration and Maintenance Periods

\begin{tabular}{|c|c|c|c|c|c|c|c|c|}
\hline & Overall & Levetiracetam & Lamotrigine & Lacosamide & Valproate & Topiramate & Carbamazepine & Phenytoin \\
\hline & $\mathrm{N}=811$ & $\mathrm{n}=156$ & $\mathrm{n}=128$ & $\mathrm{n}=125$ & $\mathrm{n}=120$ & $\mathrm{n}=114$ & $\mathrm{n}=107$ & $n=61$ \\
\hline \multicolumn{9}{|c|}{ Incidence rate of seizures (per person-month), mean (95\% CI) } \\
\hline \multicolumn{9}{|l|}{ During titration period } \\
\hline Any seizures ${ }^{\mathrm{a}}$ & $\begin{array}{c}0.320 \\
(0.294-0.349) \\
\end{array}$ & $\begin{array}{c}0.286 \\
(0.227-0.355) \\
\end{array}$ & \begin{tabular}{|c|}
0.312 \\
$(0.251-0.384)$ \\
\end{tabular} & \begin{tabular}{|c|}
0.361 \\
$(0.291-0.443)$ \\
\end{tabular} & $\begin{array}{c}0.330 \\
(0.261-0.410) \\
\end{array}$ & $\begin{array}{c}0.287 \\
(0.224-0.362)\end{array}$ & $\begin{array}{c}0.383 \\
(0.306-0.473)\end{array}$ & \begin{tabular}{|c|}
0.257 \\
$(0.165-0.382)$ \\
\end{tabular} \\
\hline $\begin{array}{l}\text { Focal aware } \\
\text { (simple partial onset) }\end{array}$ & $\begin{array}{c}0.041 \\
(0.032-0.052) \\
\end{array}$ & $\begin{array}{c}0.042 \\
(0.022-0.074) \\
\end{array}$ & \begin{tabular}{|c|}
0.028 \\
$(0.012-0.055)$ \\
\end{tabular} & $\begin{array}{c}0.094 \\
(0.060-0.140) \\
\end{array}$ & $\begin{array}{c}0.041 \\
(0.020-0.076) \\
\end{array}$ & $\begin{array}{c}0.025 \\
(0.009-0.053) \\
\end{array}$ & $\begin{array}{c}0.023 \\
(0.007-0.053) \\
\end{array}$ & $\begin{array}{c}0.021 \\
(0.003-0.077) \\
\end{array}$ \\
\hline $\begin{array}{l}\text { Focal impaired awaren } \\
\text { (complex partial onset }\end{array}$ & $\begin{array}{c}0.147 \\
(0.129-0.167) \\
\end{array}$ & $\begin{array}{c}0.162 \\
(0.119-0.216)\end{array}$ & $\begin{array}{c}0.167 \\
(0.123-0.221) \\
\end{array}$ & $\begin{array}{c}0.188 \\
(0.139-0.250) \\
\end{array}$ & $\begin{array}{c}0.152 \\
(0.107-0.210)\end{array}$ & $\begin{array}{c}0.115 \\
(0.076-0.166)\end{array}$ & $\begin{array}{c}0.108 \\
(0.069-0.161) \\
\end{array}$ & $\begin{array}{c}0.096 \\
(0.044-0.183) \\
\end{array}$ \\
\hline $\begin{array}{l}\text { Focal to bilateral tonic-clonic } \\
\text { (secondary generalized) }\end{array}$ & $\begin{array}{c}0.047 \\
(0.037-0.059) \\
\end{array}$ & $\begin{array}{c}0.032 \\
(0.015-0.060)\end{array}$ & $\begin{array}{c}0.042 \\
(0.022-0.073) \\
\end{array}$ & $\begin{array}{c}0.031 \\
(0.014-0.062) \\
\end{array}$ & $\begin{array}{c}0.066 \\
(0.038-0.107) \\
\end{array}$ & $\begin{array}{c}0.049 \\
(0.025-0.086)\end{array}$ & $\begin{array}{c}0.068 \\
(0.038-0.111)\end{array}$ & $\begin{array}{c}0.054 \\
(0.017-0.125) \\
\end{array}$ \\
\hline Generalized & $\begin{array}{c}0.076 \\
(0.063-0.091) \\
\end{array}$ & $\begin{array}{c}0.042 \\
(0.022-0.074) \\
\end{array}$ & \begin{tabular}{|c|}
0.069 \\
$(0.042-0.107)$ \\
\end{tabular} & $\begin{array}{c}0.047 \\
(0.024-0.082) \\
\end{array}$ & $\begin{array}{c}0.070 \\
(0.041-0.112) \\
\end{array}$ & $\begin{array}{c}0.078 \\
(0.047-0.122) \\
\end{array}$ & $\begin{array}{c}0.167 \\
(0.117-0.230) \\
\end{array}$ & $\begin{array}{c}0.075 \\
(0.030-0.154) \\
\end{array}$ \\
\hline Unclassified & $\begin{array}{c}0.004 \\
(0.002-0.009)\end{array}$ & $\begin{array}{c}0.007 \\
(0.001-0.025) \\
\end{array}$ & $\begin{array}{c}0.007 \\
(0.001-0.025) \\
\end{array}$ & $\begin{array}{c}0.000 \\
-\end{array}$ & $\begin{array}{c}0.000 \\
-\end{array}$ & $\begin{array}{c}0.000 \\
-\end{array}$ & $\begin{array}{r}0 . \\
(0.001 \\
\end{array}$ & $\begin{array}{c}0.011 \\
(0.000-0.060)\end{array}$ \\
\hline \multicolumn{9}{|l|}{ During maintenance period } \\
\hline Any seizures & $\begin{array}{c}0.095 \\
(0.085-0.106) \\
\end{array}$ & $\begin{array}{c}0.040 \\
(0.026-0.059) \\
\end{array}$ & \begin{tabular}{|c|}
0.077 \\
$(0.054-0.107)$ \\
\end{tabular} & $\begin{array}{c}0.156 \\
(0.123-0.194) \\
\end{array}$ & $\begin{array}{c}0.108 \\
(0.081-0.142) \\
\end{array}$ & $\begin{array}{c}0.094 \\
(0.068-0.128) \\
\end{array}$ & $\begin{array}{c}0.144 \\
(0.110-0.185) \\
\end{array}$ & $\begin{array}{c}0.041 \\
(0.021-0.074) \\
\end{array}$ \\
\hline $\begin{array}{l}\text { Focal a } \\
\text { (simple }\end{array}$ & $\begin{array}{c}0.007 \\
(0.004-0.010)\end{array}$ & $\begin{array}{c}0.000 \\
-\end{array}$ & $\begin{array}{c}0.006 \\
(0.001-0.018) \\
\end{array}$ & $\begin{array}{c}0.010 \\
(0.003-0.023)\end{array}$ & $\begin{array}{c}0.012 \\
(0.005-0.027)\end{array}$ & $\begin{array}{c}0.007 \\
(0.001-0.020) \\
\end{array}$ & $\begin{array}{c}0.009 \\
(0.003-0.024) \\
\end{array}$ & $\begin{array}{c}0.000 \\
-\end{array}$ \\
\hline $\begin{array}{l}\text { Focal impa } \\
\text { (complex p }\end{array}$ & $\begin{array}{c}0.033 \\
(0.027-0.040) \\
\end{array}$ & $\begin{array}{c}0.021 \\
(0.011-0.036) \\
\end{array}$ & \begin{tabular}{|c|}
0.044 \\
$(0.027-0.067)$ \\
\end{tabular} & $\begin{array}{c}0.046 \\
(0.029-0.069) \\
\end{array}$ & $\begin{array}{c}0.027 \\
(0.014-0.046) \\
\end{array}$ & $\begin{array}{c}0.046 \\
(0.028-0.071)\end{array}$ & $\begin{array}{c}0.031 \\
(0.016-0.052) \\
\end{array}$ & $\begin{array}{c}0.015 \\
(0.004-0.038) \\
\end{array}$ \\
\hline $\begin{array}{l}\text { Focal to bilat } \\
\text { (secondary g }\end{array}$ & $\begin{array}{c}0.025 \\
(0.020-0.031) \\
\end{array}$ & $\begin{array}{c}0.003 \\
(0.000-0.012) \\
\end{array}$ & $\begin{array}{c}0.017 \\
(0.007-0.033) \\
\end{array}$ & $\begin{array}{c}0.080 \\
(0.057-0.109) \\
\end{array}$ & $\begin{array}{c}0.017 \\
(0.007-0.033) \\
\end{array}$ & $\begin{array}{c}0.023 \\
(0.011-0.042)\end{array}$ & $\begin{array}{c}0.021 \\
(0.010-0.040)\end{array}$ & $\begin{array}{c}0.011 \\
(0.002-0.033) \\
\end{array}$ \\
\hline Generalized & $\begin{array}{c}0.023 \\
(0.018-0.029)\end{array}$ & $\begin{array}{c}0.014 \\
(0.007-0.027)\end{array}$ & $\begin{array}{c}0.008 \\
(0.002-0.021)\end{array}$ & $\begin{array}{c}0.016 \\
(0.007-0.031)\end{array}$ & $\begin{array}{c}0.027 \\
(0.014-0.046)\end{array}$ & $\begin{array}{c}0.011 \\
(0.004-0.027)\end{array}$ & $\begin{array}{c}0.080 \\
(0.055-0.112)\end{array}$ & $\begin{array}{c}0.007 \\
(0.001-0.027)\end{array}$ \\
\hline Uncla & $\begin{array}{c}0.002 \\
(0.001-0.004) \\
\end{array}$ & $\begin{array}{c}0.002 \\
(0.000-0.009)\end{array}$ & $\begin{array}{c}0.002 \\
(0.000-0.012) \\
\end{array}$ & $\begin{array}{c}0.004 \\
(0.000-0.014) \\
\end{array}$ & $\begin{array}{c}0.000 \\
- \\
\end{array}$ & $\begin{array}{c}0.000 \\
- \\
\end{array}$ & $\begin{array}{r}0 . \\
(0.000 \\
\end{array}$ & $\begin{array}{c}0.004 \\
(0.000-0.021) \\
\end{array}$ \\
\hline \multicolumn{9}{|c|}{ Incidence rate of HRU (per person-month) } \\
\hline \multicolumn{9}{|l|}{ During titration period } \\
\hline Hospitalization & $\begin{array}{c}0.018 \\
(0.012-0.026) \\
\end{array}$ & $\begin{array}{c}0.011 \\
(0.002-0.031) \\
\end{array}$ & \begin{tabular}{|c|}
0.014 \\
$(0.004-0.036)$ \\
\end{tabular} & $\begin{array}{c}0.016 \\
(0.004-0.040) \\
\end{array}$ & $\begin{array}{c}0.004 \\
(0.000-0.023) \\
\end{array}$ & $\begin{array}{c}0.012 \\
(0.003-0.036) \\
\end{array}$ & $\begin{array}{c}0.045 \\
(0.022-0.083) \\
\end{array}$ & $\begin{array}{c}0.043 \\
(0.012-0.110) \\
\end{array}$ \\
\hline Emergency de & $\begin{array}{c}0.034 \\
(0.026-0.045) \\
\end{array}$ & $\begin{array}{c}0.025 \\
(0.010-0.051) \\
\end{array}$ & \begin{tabular}{|c|}
0.035 \\
$(0.017-0.064)$ \\
\end{tabular} & $\begin{array}{c}0.043 \\
(0.022-0.077) \\
\end{array}$ & $\begin{array}{c}0.029 \\
(0.012-0.059) \\
\end{array}$ & $\begin{array}{c}0.016 \\
(0.004-0.042)\end{array}$ & $\begin{array}{c}0.041 \\
(0.019-0.077) \\
\end{array}$ & $\begin{array}{c}0.086 \\
(0.037-0.169) \\
\end{array}$ \\
\hline Outp & $\begin{array}{c}0.312 \\
(0.286-0.341) \\
\end{array}$ & $\begin{array}{c}0.247 \\
(0.192-0.312) \\
\end{array}$ & $\begin{array}{c}0.278 \\
(0.220-0.345) \\
\end{array}$ & $\begin{array}{c}0.259 \\
(0.200-0.329) \\
\end{array}$ & $\begin{array}{c}0.437 \\
(0.358-0.528) \\
\end{array}$ & $\begin{array}{c}0.266 \\
(0.205-0.339)\end{array}$ & $\begin{array}{c}0.441 \\
(0.358-0.538)\end{array}$ & $\begin{array}{c}0.257 \\
(0.165-0.382) \\
\end{array}$ \\
\hline Referral to otl & $\begin{array}{c}0.013 \\
(0.008-0.020) \\
\end{array}$ & $\begin{array}{c}0.007 \\
(0.001-0.025) \\
\end{array}$ & $\begin{array}{c}0.014 \\
(0.004-0.036) \\
\end{array}$ & $\begin{array}{c}0.016 \\
(0.004-0.040) \\
\end{array}$ & $\begin{array}{c}0.008 \\
(0.001-0.030) \\
\end{array}$ & $\begin{array}{c}0.000 \\
-\end{array}$ & $\begin{array}{c}0.027 \\
(0.010-0.059) \\
\end{array}$ & $\begin{array}{c}0.032 \\
(0.007-0.094) \\
\end{array}$ \\
\hline $\begin{array}{l}\text { Order for laboratory testing and/ } \\
\text { or diagnostic imaging }\end{array}$ & $\begin{array}{c}0.279 \\
(0.254-0.306)\end{array}$ & $\begin{array}{c}0.317 \\
(0.255-0.390)\end{array}$ & $\begin{array}{c}0.198 \\
(0.150-0.256) \\
\end{array}$ & $\begin{array}{c}0.275 \\
(0.214-0.347) \\
\end{array}$ & $\begin{array}{c}0.309 \\
(0.243-0.387)\end{array}$ & $\begin{array}{c}0.193 \\
(0.141-0.256)\end{array}$ & $\begin{array}{c}0.297 \\
(0.230-0.378) \\
\end{array}$ & $\begin{array}{c}0.535 \\
(0.397-0.705) \\
\end{array}$ \\
\hline Phone call to office personnel & $\begin{array}{c}0.293 \\
(0.268-0.321) \\
\end{array}$ & $\begin{array}{c}0.296 \\
(0.236-0.367) \\
\end{array}$ & \begin{tabular}{|c|}
0.271 \\
$(0.214-0.338)$ \\
\end{tabular} & $\begin{array}{c}0.349 \\
(0.280-0.430) \\
\end{array}$ & $\begin{array}{c}0.309 \\
(0.243-0.387) \\
\end{array}$ & $\begin{array}{c}0.340 \\
(0.271-0.421) \\
\end{array}$ & $\begin{array}{c}0.216 \\
(0.159-0.287) \\
\end{array}$ & $\begin{array}{c}0.225 \\
(0.139-0.344) \\
\end{array}$ \\
\hline \multicolumn{9}{|l|}{ During maintenance period } \\
\hline Hospitalization & $\begin{array}{c}0.008 \\
(0.005-0.012) \\
\end{array}$ & $\begin{array}{c}0.006 \\
(0.002-0.016)\end{array}$ & $\begin{array}{c}0.004 \\
(0.001-0.015) \\
\end{array}$ & $\begin{array}{c}0.004 \\
(0.000-0.014) \\
\end{array}$ & $\begin{array}{c}0.006 \\
(0.001-0.018) \\
\end{array}$ & $\begin{array}{c}0.009 \\
(0.003-0.023)\end{array}$ & $\begin{array}{c}0.019 \\
(0.008-0.037) \\
\end{array}$ & $\begin{array}{c}0.011 \\
(0.002-0.033) \\
\end{array}$ \\
\hline Emergency department visit ${ }^{b}$ & $\begin{array}{c}0.016 \\
(0.012-0.021) \\
\end{array}$ & $\begin{array}{c}0.011 \\
(0.005-0.023)\end{array}$ & \begin{tabular}{|c|}
0.006 \\
$(0.001-0.018)$ \\
\end{tabular} & $\begin{array}{c}0.012 \\
(0.004-0.026) \\
\end{array}$ & $\begin{array}{c}0.027 \\
(0.014-0.046) \\
\end{array}$ & $\begin{array}{c}0.016 \\
(0.006-0.033) \\
\end{array}$ & $\begin{array}{c}0.021 \\
(0.010-0.040) \\
\end{array}$ & $\begin{array}{c}0.019 \\
(0.006-0.044) \\
\end{array}$ \\
\hline Outpatient visit & $\begin{array}{c}0.236 \\
(0.219-0.253) \\
\end{array}$ & $\begin{array}{c}0.233 \\
(0.197-0.275) \\
\end{array}$ & $\begin{array}{c}0.188 \\
(0.151-0.231) \\
\end{array}$ & $\begin{array}{c}0.251 \\
(0.209-0.299) \\
\end{array}$ & $\begin{array}{c}0.265 \\
(0.221-0.315) \\
\end{array}$ & $\begin{array}{c}0.241 \\
(0.197-0.292)\end{array}$ & $\begin{array}{c}0.233 \\
(0.190-0.284) \\
\end{array}$ & $\begin{array}{c}0.240 \\
(0.185-0.306) \\
\end{array}$ \\
\hline Referral to other physician & $\begin{array}{c}0.004 \\
(0.002-0.007) \\
\end{array}$ & $\begin{array}{c}0.005 \\
(0.001-0.014) \\
\end{array}$ & $\begin{array}{c}0.000 \\
-\end{array}$ & $\begin{array}{c}0.010 \\
(0.003-0.023) \\
\end{array}$ & $\begin{array}{c}0.002 \\
(0.000-0.012)\end{array}$ & $\begin{array}{c}0.007 \\
(0.001-0.020)\end{array}$ & $\begin{array}{c}0.005 \\
(0.001-0.017) \\
\end{array}$ & $\begin{array}{c}0.000 \\
-\end{array}$ \\
\hline $\begin{array}{l}\text { Order for laboratory testing and/ } \\
\text { or diagnostic imaging }\end{array}$ & $\begin{array}{c}0.030 \\
(0.024-0.036) \\
\end{array}$ & $\begin{array}{c}0.016 \\
(0.008-0.029)\end{array}$ & $\begin{array}{c}0.021 \\
(0.010-0.038) \\
\end{array}$ & $\begin{array}{c}0.030 \\
(0.017-0.049) \\
\end{array}$ & $\begin{array}{c}0.035 \\
(0.021-0.057) \\
\end{array}$ & $\begin{array}{c}0.041 \\
(0.024-0.065)\end{array}$ & $\begin{array}{c}0.042 \\
(0.025-0.067) \\
\end{array}$ & $\begin{array}{c}0.030 \\
(0.013-0.059) \\
\end{array}$ \\
\hline Phone call to office personnel & $\begin{array}{c}0.038 \\
(0.032-0.046) \\
\end{array}$ & $\begin{array}{c}0.026 \\
(0.015-0.042)\end{array}$ & $\begin{array}{c}0.048 \\
(0.030-0.072) \\
\end{array}$ & $\begin{array}{c}0.052 \\
(0.034-0.076) \\
\end{array}$ & $\begin{array}{c}0.031 \\
(0.017-0.052) \\
\end{array}$ & $\begin{array}{c}0.067 \\
(0.045-0.096) \\
\end{array}$ & $\begin{array}{c}0.028 \\
(0.015-0.049) \\
\end{array}$ & $\begin{array}{c}0.007 \\
(0.001-0.027) \\
\end{array}$ \\
\hline
\end{tabular}

aSeizure classifications are based on the 2017 ILAE classifications ${ }^{5}$; previous 1981 classifications are in parentheses. ${ }^{23}$

${ }^{b}$ Emergency department visit included visits that resulted in a hospitalization. These visits were counted as an emergency department visit and a hospitalization. $C I=$ confidence interval; $H R U=$ health care resource use; ILAE = International League Against Epilepsy. 


\section{FIGURE 2 Adjusted Incidence Rate Ratios of Seizures and Epilepsy-Related Health Care Resource Use During} Titration Versus Maintenance

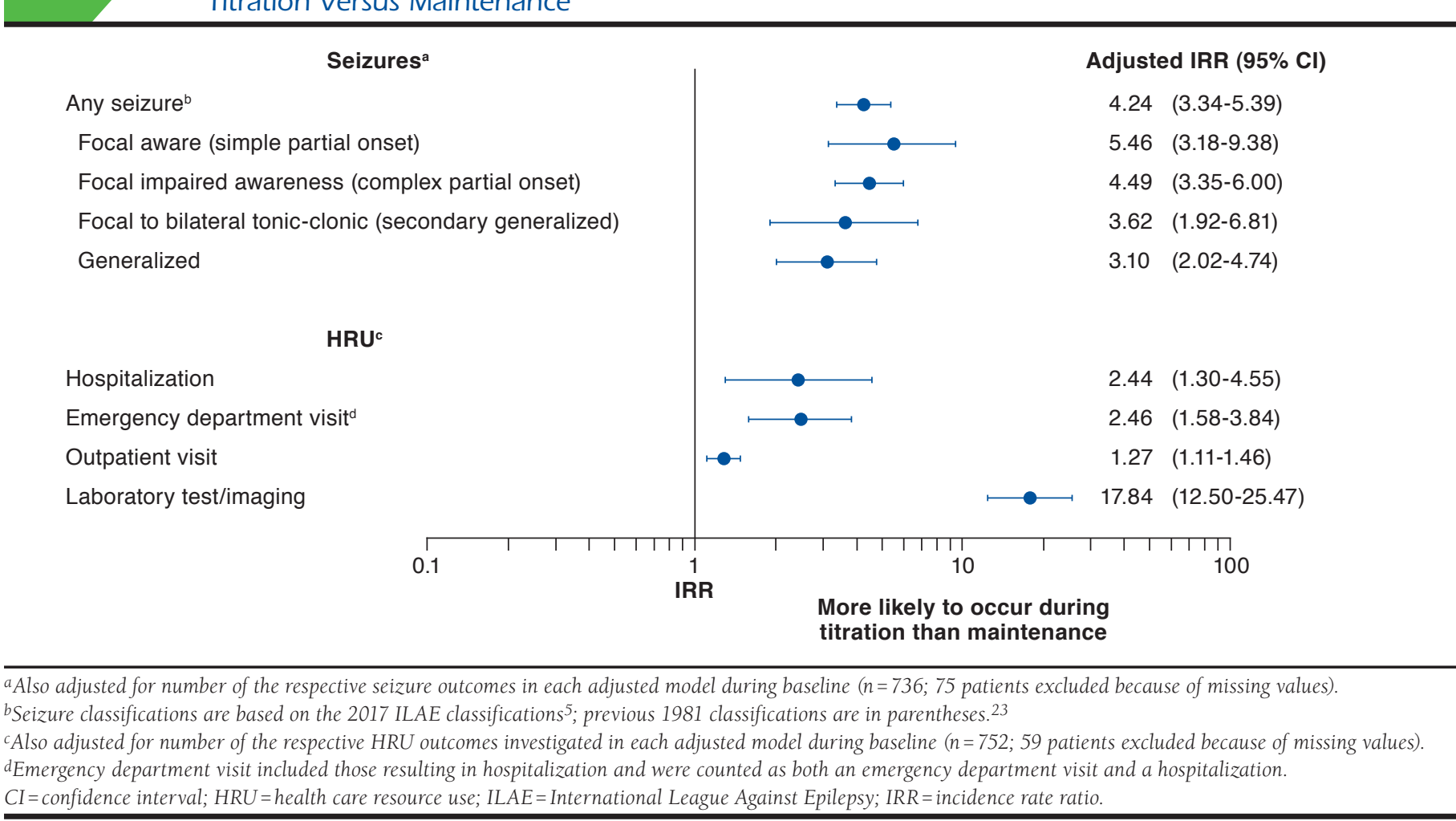

titration (adjusted cost ratio of 1.05 [95\% CI $=1.01-1.09]$ ). Costs associated with each HRU type decreased from titration to maintenance period; total health care costs per person-month decreased by $46.9 \%$ (Figure 3). Comparison of costs during titration versus maintenance resulted in adjusted cost ratios of 2.67 (95\% CI $=2.06-3.46)$ for total costs, $2.77(95 \% \mathrm{CI}=1.57-4.89)$ for emergency department visits, 10.51 (95\% CI=7.60-14.53) for laboratory testing/imaging, 1.52 (95\% CI=0.60-3.84) for hospitalizations, and 1.05 (95\% CI=0.87-1.26) for outpatient costs. Fifty-nine patients were excluded from the epilepsyrelated HRU models due to missing covariates.

\section{Discussion}

This study described the time from AED initiation to physician-assessed maintenance dose (i.e., titration duration) for AEDs in real-world clinical practice and assessed clinical outcomes, HRU, and costs associated with titration of newly initiated AED treatment. Patient epilepsy and treatment history (i.e., $<1$ year since diagnosis, $42 \%$ without prior AED treatment, $77 \%$ with no treatment on index date) indicates a population with less severe epilepsy who may respond to AED treatment. Studies have shown that up to two thirds of previously untreated patients will attain freedom from seizures with first AED monotherapy. ${ }^{27}$ Compared with maintenance, AED titration was associated with a 3- to 5.5-fold higher rate of seizures as well as $>2$-fold higher rates of emergency department visits and hospitalization. Incidence rates of HRU decreased from titration to maintenance, with 54.5\%-89.3\% lower incidences observed for all HRU categories except outpatient visits (24.6\% decrease). Total epilepsy-related monthly costs decreased $46.9 \%$ from titration to maintenance.

It should be noted that use of a previous study to derive unit costs led to a conservative estimate of HRU cost. ${ }^{24}$ Our assessment of costs during the 6-month period after new treatment initiation may have implications for insurance providers, as a majority of commercially insured patients $\left(82.7 \%\right.$ in $\left.2015^{28}\right)$ will not change plans, insurers, or exit commercial insurance in a given year, potentially before treatment benefit and associated savings are realized. The current study suggests that potential costs associated with titration would likely be incurred by a health plan soon after AED initiation. To our knowledge, this is the first study that examined the costs of AED titration and found that it is an underrecognized source of high health care costs.

Previous studies have reported increased HRU and costs associated with uncontrolled versus controlled seizures ${ }^{29}$ and with AED nonadherence versus adherence. ${ }^{30}$ However, the association of HRU and costs during the time frame when 


\section{FIGURE 3 Overall Health Care Resource Use Costs per Person-Month During Titration} and Maintenance $(\mathrm{N}=811)^{\mathrm{a}}$

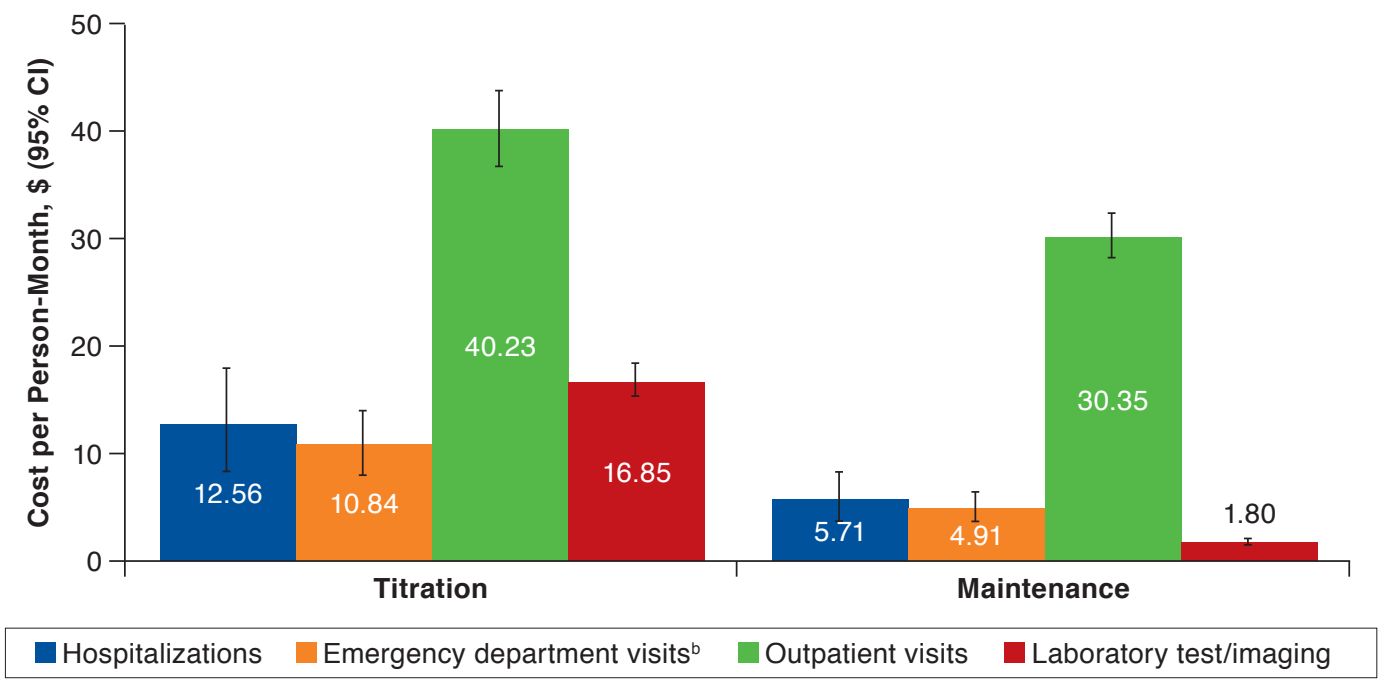

a2016 U.S. dollars.

${ }^{b}$ Emergency department visits included visits that resulted in a hospitalization. These visits were counted as both an emergency department visit and a hospitalization. $C I=$ confidence interval.

patients are being titrated to an effective yet tolerated AED dose has not been previously reported. The association of the titration period with higher HRU rates and costs versus maintenance parallels reports of titration periods in other disease states, such as selective serotonin reuptake inhibitors in major depression, ${ }^{31}$ where delay in therapeutic benefit leads to continued resource use. In our study, increased HRU and costs may be partly attributed to the higher risk of seizures reported during the titration period.

Initiating AED therapy at a low dose and titrating over a period of weeks is often appropriate to minimize potential side effects while reaching a minimally effective dose, as quality of life suffers when patients experience AED-related adverse events. ${ }^{32}$ However, more rapid titration may be necessary for patients with recent recurrent seizures or acute severe seizure events. Patients who are high risk should be considered for immediate AED treatment and may need rapid dose escalation. ${ }^{33}$ Newer AEDs are often associated with improved safety and efficacy compared with older AEDs, and the advent of AEDs with a shorter-to-no titration requirement will expand therapeutic options.

\section{Limitations}

As with any retrospective observational study, there is the potential for missing or inaccurate data recorded in the primary medical charts, or for errors introduced during data entry. Encounters separate from the primary treating physician's network may not have been captured. Further, because patients may see their physician less frequently during AED maintenance, it is possible that seizure frequency was underreported during the maintenance period. However, the study required that the number of patient-reported seizures between clinical visits is available in the medical record in order to mitigate the underreporting of seizure frequency during periods with less frequent physician visits.

Patients with missing baseline covariates (approximately $7 \%-9 \%$ of patients) were excluded from the adjusted multivariable analyses. Physicians were instructed to select patients with complete medical records during the study period, and realtime error and logic checking were implemented in the case report form to mitigate this limitation. In addition, detailed guidance for random patient selection was provided to the physicians to avoid selecting patients based on any study outcomes and to reduce selection bias.

When looking at trends in AED use, it should be noted that not all AEDs are appropriate for each seizure type. Despite efforts to ensure that similar proportions of patients initiating various AEDs were enrolled, some AEDs, such as phenytoin, were relatively underrepresented, which may reflect a less frequent use in clinical practice.

Disease severity may factor into overall cost and was not directly assessed; however, proxies for severity, such as 
epilepsy history, seizure frequency, and prior treatment, were collected. Moreover, health care costs were not estimated from the actual medical service cost of the patients but derived from the incidence rate and unit costs of HRU. Reporting HRU instead of costs allows a health plan the ability to remove cost variability and negotiated rates and better assess resource use. Because HRU costs were based on an older study and adjusted to 2016 U.S. dollars, they may underestimate current health care costs. Finally, patient adherence to prescribed therapies cannot be verified from chart review.

\section{Conclusions}

Our study demonstrated that AED titration is costly, with increased HRU rates and costs during AED titration versus maintenance. In addition, a 5\% increase in total costs per additional month of titration was observed. Combined, these data suggest that the sooner patients reach a therapeutic maintenance dose and attain seizure control, the sooner patientspecific benefits (i.e., fewer seizures, less HRU, and lower costs) may be realized.

It should be noted that other factors (e.g., patient response to an AED, HRU associated with AED titration [e.g., monitoring for some AEDs]) will also affect the attainment of patientspecific benefits. Costs of titration are seen within a short time period, and medications that optimize this period may benefit the patient. The introduction of newer AEDs with therapeutically effective initiation doses may affect outcomes, and it will be of interest for future research to investigate whether HRU patterns and costs differ between AEDs without titration and those requiring titration.

\section{Authors}

JESSE FISHMAN, PharmD, and IMANE WILD, PhD, UCB Pharma, Smyrna, Georgia. LINDA KALILANI, PhD, UCB Pharma, Raleigh, North Carolina. YAN SONG, PhD, and ELYSE SWALLOW, MPP, Analysis Group, Boston, Massachusetts.

AUTHOR CORRESPONDENCE: Jesse Fishman, PharmD, UCB Pharma, 1950 Lake Park Dr. SE, Smyrna, GA 30080. Tel.: 770.970.8823; E-mail: jesse.fishman@ucb.com.

\section{DISCLOSURES}

UCB Pharma sponsored this study and reviewed the manuscript. Fishman and Kalilani are employees of UCB Pharma. Wild was an employee of UCB Pharma at the time this analysis was conducted. Song and Swallow are employees of Analysis Group, which received funding from UCB Pharma.

\section{ACKNOWLEDGMENTS}

The authors thank the physicians who provided patient case information for this study. The authors acknowledge Lynne Isbell, PhD, CMPP (Evidence Scientific Solutions, Philadelphia, PA) for writing assistance, which was funded by UCB Pharma.

\section{REFERENCES}

1. Zack MM, Kobau R. National and state estimates of the numbers of adults and children with active epilepsy-United States, 2015. MMWR Morb Mortal Wkly Rep. 2017;66(31):821-25.

2. World Health Organization. Epilepsy fact sheet. 2017. Available at: http:// www.who.int/mediacentre/factsheets/fs999/en/. Accessed February 3, 2018.

3. Fazel S, Wolf A, Långström N, Newton CR, Lichtenstein P. Premature mortality in epilepsy and the role of psychiatric comorbidity: a total population study. Lancet. 2013;382(9905):1646-54

4. Thurman DJ, Logroscino G, Beghi E, et al. The burden of premature mortality of epilepsy in high-income countries: a systematic review from the Mortality Task Force of the International League Against Epilepsy. Epilepsia. 2017;58(1):17-26.

5. Fisher RS, Cross JH, French JA, et al. Operational classification of seizure types by the International League Against Epilepsy: position paper of the ILAE Commission for Classification and Terminology. Epilepsia. 2017:58(4):522-30.

6. Sander JW. New antiepileptic drugs in practice--how do they perform in the real world? Acta Neurol Scand Suppl. 2005;181:26-29.

7. Sander JW. The use of antiepileptic drugs-principles and practice. Epilepsia. 2004:45 Suppl 6:28-34.

8. Shorvon SD. The Antiepileptic Drugs. Handbook of Epilepsy Treatment. Chichester, West Sussex, UK: Wiley-Blackwell; 2010

9. Glauser T, Ben-Menachem E, Bourgeois B, et al. ILAE treatment guidelines: evidence-based analysis of antiepileptic drug efficacy and effectiveness as initial monotherapy for epileptic seizures and syndromes. Epilepsia. 2006;47(7):1094-120

10. Treiman DM. Management of refractory complex partial seizures: current state of the art. Neuropsychiatr Dis Treat. 2010;6:297-308.

11. National Institute for Health and Care Excellence. Epilepsies: diagnosis and management. Clinical guideline [CG137]. 2012, updated 2016. Available at: https://www.nice.org.uk/guidance/cg137. Accessed February 3, 2018.

12. Panayiotopoulos CP. Chapter 4, Principles of therapy in epilepsies. In Panayiotopoulos CP. The Epilepsies: Seizures, Syndromes and Management. Oxfordshire, UK: Bladon Medical Publishing; 2005. Available at: https:// www.ncbi.nlm.nih.gov/books/NBK2607/\#ch4.sl. Accessed February 5, 2018. 13. Lamictal (lamotrigine) for oral use. GlaxoSmithKline. 2015. Available at: http://www.accessdata.fda.gov/drugsatfda_docs/label/2015/02024ls053,020 764s046,022251s0171bl.pdf. Accessed February 3, 2018

14. Epilepsy Foundation. Algorithm for treating epilepsy patients with valproate (Depakote, valproic acid). Available at: http://www.epilepsy.com/sites/ core/files/atoms/files/epilepsy_valproate_0.pdf. Accessed February 3, 2018.

15. Sirven JI, Krauss GL, Chung S. To titrate or not: optimizing treatment with antiepileptic drugs. 2015. Available at: http://www.medscape.org/viewarticle/848142_transcript_2. Accessed February 3, 2018.

16. Biton V, Edwards KR, Montouris GD, et al. Topiramate titration and tolerability. Ann Pharmacother. 2001;35(2):173-79.

17. Sander JW. Overview of established antiepileptic drugs. In: Rugg-Gunn FJ, Smalls JE, eds. Epilepsy 2015: From Channels to Commissioning. A Practical Guide to Epilepsy. West Hartford, CT: International League Against Epilepsy; 2015:299-316. Available at: https://www.epilepsysociety.org.uk/sites/default/ files/attachments/Chapter28Sander2015.pdf. Accessed February 13, 2018.

18. Makin C. Comparison of health care resource utilization and costs in treatment-adherent patients with epilepsy with and without breakthrough seizures in a U.S. managed care population. Neurology. 2014; 82(10 Suppl):P5.069.

19. Warshavsky A, Eilam A, Gilad R. Lamotrigine as monotherapy in clinical practice: efficacy of various dosages in epilepsy. Brain Behav. 2016;6(3):e00419. 
20. Divino V, Petrilla AA, Bollu V, Velez F, Ettinger A, Makin C. Clinical and economic burden of breakthrough seizures. Epilepsy Behav. 2015;51:40-47.

21. Ryvlin P, Werhahn KJ, Blaszczyk B, Johnson ME, Lu S. Adjunctive brivaracetam in adults with uncontrolled focal epilepsy: results from a doubleblind, randomized, placebo-controlled trial. Epilepsia. 2014;55(1):47-56.

22. Quan H, Li B, Couris CM, et al. Updating and validating the Charlson comorbidity index and score for risk adjustment in hospital discharge abstracts using data from 6 countries. Am J Epidemiol. 2011;173(6):676-82.

23. Proposal for revised clinical and electroencephalographic classification of epileptic seizures. From the Commission on Classification and Terminology of the International League Against Epilepsy. Epilepsia. 1981;22(4):489-501.

24. Begley CE, Lairson DR, Reynolds TF, Coan S. Early treatment cost in epilepsy and how it varies with seizure type and frequency. Epilepsy Res. 2001:47(3):205-15.

25. Rothman KJ, Greenland S, Lash TL. Modern Epidemiology. Philadelphia, PA: Lippincott Williams \& Wilkins; 2008.

26. Lauderdale BE. Compound Poisson-Gamma regression models for dollar outcomes that are sometimes zero. Polit Anal. 2012;20(3):387-99.

27. Brodie MJ, Barry SJ, Bamagous GA, Norrie JD, Kwan P. Patterns of treatment response in newly diagnosed epilepsy. Neurology. 2012;78(20):1548-54.
28. Sung I. ACAView research brief: the impact of health care reform on insurance switching patterns. July 16, 2015. Available at: https://www.athenahealth.com/blog/2015/07/16/acaview-research-brief-the-impact-of-healthcare-reform-on-insurance-switching-patterns. Accessed February 3, 2018.

29. Cramer JA, Wang ZJ, Chang E, et al. Healthcare utilization and costs in adults with stable and uncontrolled epilepsy. Epilepsy Behav. 2014;31:356-62.

30. Faught RE, Weiner JR, Guérin A, Cunnington MC, Duh MS. Impact of nonadherence to antiepileptic drugs on health care utilization and costs: findings from the RANSOM study. Epilepsia. 2009;50(3):501-09.

31. Camacho F, Kong MC, Sheehan DV, Balkrishnan R. Expenditures associated with dose titration at initiation of therapy in patients with major depressive disorder: a retrospective analysis of a large managed care claims database. P T. 2010;35(8):452-68.

32. Louis EK. The art of managing conversions between antiepileptic drugs: maximizing patient tolerability and quality of life. Pharmaceuticals (Basel). 2010;3(9):2956-69.

33. Krumholz A, Wiebe S, Gronseth GS, et al. Evidence-based guideline: management of an unprovoked first seizure in adults: report of the Guideline Development Subcommittee of the American Academy of Neurology and the American Epilepsy Society. Neurology. 2015;84(16):1705-13. 
APPENDIX Total Epilepsy-Related Health Care Resource Use Costs per Person-Month for Individual AED Groups During Titration and Maintenance Periods ${ }^{\mathrm{a}}$
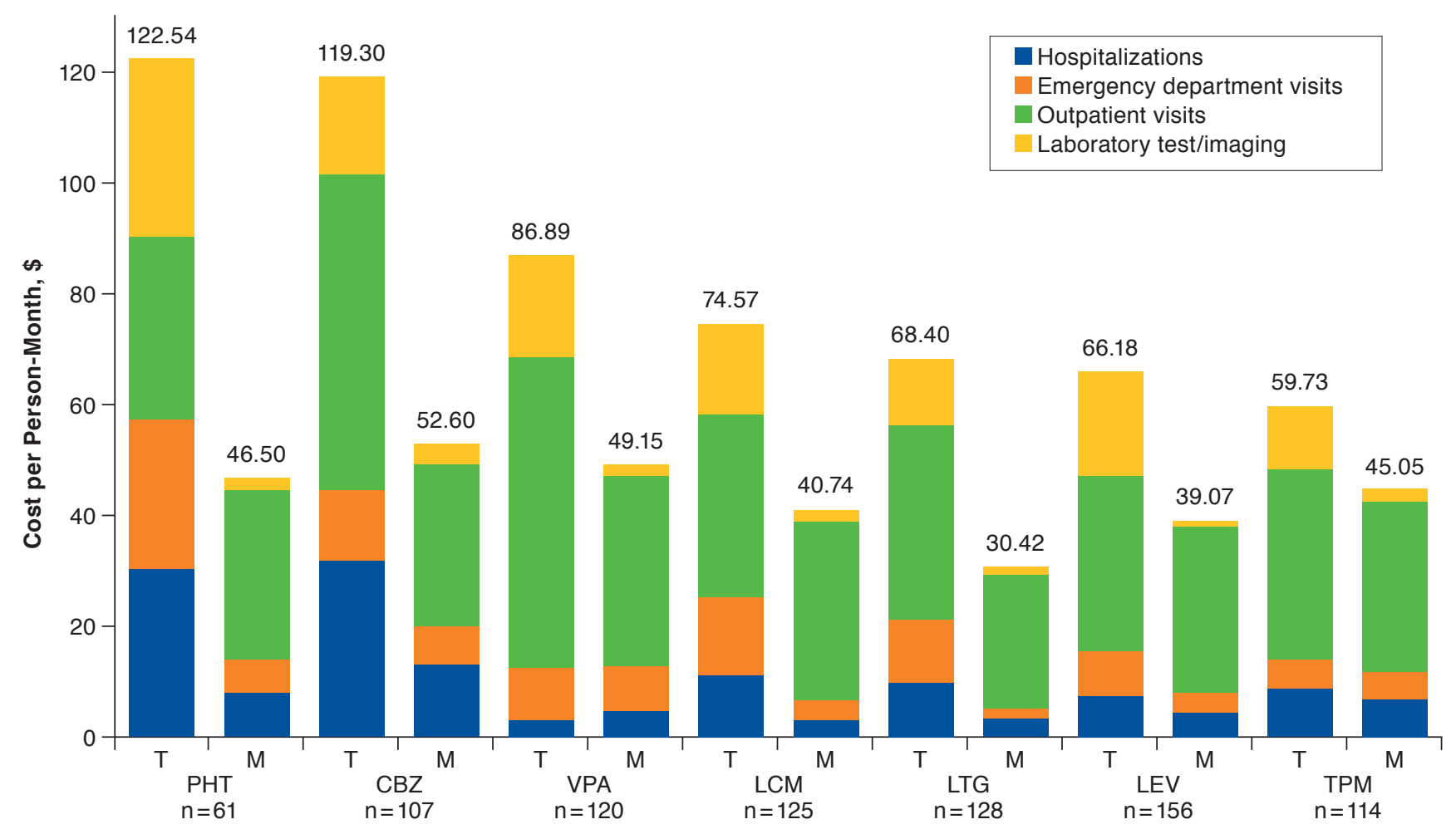

Health Care Resource Use-Specific Costs per Person-Month During Titration and Maintenance Periods for Individual AEDs (N=811)

\begin{tabular}{|c|c|c|c|c|c|c|c|c|c|c|c|c|c|c|}
\hline \multirow{2}{*}{$\begin{array}{l}2016 \text { USD, } \\
\text { mean }(95 \% \mathrm{CI})\end{array}$} & \multicolumn{2}{|c|}{$\begin{array}{c}\text { PHT } \\
\mathrm{n}=61\end{array}$} & \multicolumn{2}{|c|}{$\begin{array}{c}\text { CBZ } \\
\mathrm{n}=107\end{array}$} & \multicolumn{2}{|c|}{$\begin{array}{c}\text { VPA } \\
\mathrm{n}=120\end{array}$} & \multicolumn{2}{|c|}{$\begin{array}{c}\text { LCM } \\
\mathrm{n}=125\end{array}$} & \multicolumn{2}{|c|}{$\begin{array}{c}\text { LTG } \\
\mathrm{n}=128\end{array}$} & \multicolumn{2}{|c|}{$\begin{array}{c}\text { LEV } \\
\mathrm{n}=156\end{array}$} & \multicolumn{2}{|c|}{$\begin{array}{c}\text { TPM } \\
\mathbf{n}=114\end{array}$} \\
\hline & $\mathrm{T}$ & M & $\mathrm{T}$ & $\mathbf{M}$ & $\mathrm{T}$ & M & $\mathrm{T}$ & M & $\mathrm{T}$ & M & $\mathrm{T}$ & $\mathbf{M}$ & $\mathrm{T}$ & $\mathbf{M}$ \\
\hline Hospitalizations & $\begin{array}{l}30.21 \\
(8.23- \\
77.35)\end{array}$ & $\begin{array}{c}7.93 \\
(1.64- \\
23.17)\end{array}$ & $\begin{array}{c}31.78 \\
(15.24- \\
58.44)\end{array}$ & $\begin{array}{l}13.31 \\
(5.75- \\
26.22)\end{array}$ & $\begin{array}{c}2.91 \\
(0.07- \\
16.20)\end{array}$ & $\begin{array}{c}4.41 \\
(0.91- \\
12.89) \\
\end{array}$ & $\begin{array}{l}11.07 \\
(3.02- \\
28.35)\end{array}$ & $\begin{array}{c}2.81 \\
(0.34- \\
10.17)\end{array}$ & $\begin{array}{c}9.79 \\
(2.67- \\
25.08)\end{array}$ & $\begin{array}{c}2.95 \\
(0.36- \\
10.66)\end{array}$ & $\begin{array}{c}7.47 \\
(1.54- \\
21.82)\end{array}$ & $\begin{array}{c}4.51 \\
(1.23- \\
11.56)\end{array}$ & $\begin{array}{c}8.67 \\
(1.79- \\
25.34)\end{array}$ & $\begin{array}{r}6.48 \\
(1.76- \\
16.58)\end{array}$ \\
\hline $\begin{array}{l}\text { Emergency } \\
\text { department } \\
\text { visitsb }^{\text {t }}\end{array}$ & $\begin{array}{l}26.99 \\
(11.65- \\
53.18)\end{array}$ & $\begin{array}{c}5.90 \\
(1.92- \\
13.78)\end{array}$ & $\begin{array}{l}12.78 \\
(5.84- \\
24.25)\end{array}$ & $\begin{array}{c}6.69 \\
(3.06- \\
12.69)\end{array}$ & $\begin{array}{c}9.09 \\
(3.66- \\
18.74)\end{array}$ & $\begin{array}{c}8.54 \\
(4.55- \\
14.60)\end{array}$ & $\begin{array}{l}13.60 \\
(6.79- \\
24.34)\end{array}$ & $\begin{array}{c}3.77 \\
(1.38- \\
8.21)\end{array}$ & $\begin{array}{l}10.94 \\
(5.24- \\
20.11)\end{array}$ & $\begin{array}{c}1.98 \\
(0.41- \\
5.78)\end{array}$ & $\begin{array}{c}7.78 \\
(3.13- \\
16.04)\end{array}$ & $\begin{array}{c}3.53 \\
(1.42- \\
7.27)\end{array}$ & $\begin{array}{c}5.17 \\
(1.41- \\
13.22)\end{array}$ & $\begin{array}{l}5.06 \\
(2.04- \\
10.43)\end{array}$ \\
\hline Outpatient visits & $\begin{array}{c}33.07 \\
(21.19- \\
49.21)\end{array}$ & $\begin{array}{c}30.86 \\
(23.77- \\
39.41)\end{array}$ & $\begin{array}{c}56.82 \\
(46.13- \\
69.25)\end{array}$ & $\begin{array}{l}30.05 \\
(24.42- \\
36.58)\end{array}$ & $\begin{array}{c}56.25 \\
(46.05- \\
68.03)\end{array}$ & $\begin{array}{c}34.07 \\
(28.40- \\
40.53) \\
\end{array}$ & $\begin{array}{c}33.34 \\
(25.78- \\
42.41)\end{array}$ & $\begin{array}{c}32.35 \\
(26.95- \\
38.52)\end{array}$ & $\begin{array}{c}35.74 \\
(28.34- \\
44.48)\end{array}$ & $\begin{array}{c}24.23 \\
(19.48- \\
29.78)\end{array}$ & $\begin{array}{c}31.79 \\
(24.78- \\
40.16)\end{array}$ & $\begin{array}{c}30.07 \\
(25.39- \\
35.36)\end{array}$ & $\begin{array}{c}34.28 \\
(26.46- \\
43.70)\end{array}$ & $\begin{array}{r}31.02 \\
(25.37 \\
37.55) \\
\end{array}$ \\
\hline $\begin{array}{l}\text { Laboratory } \\
\text { testing/imaging }\end{array}$ & $\begin{array}{c}32.27 \\
(23.95- \\
42.54) \\
\end{array}$ & $\begin{array}{c}1.81 \\
(0.78- \\
3.56) \\
\end{array}$ & $\begin{array}{l}17.92 \\
(13.86- \\
22.80) \\
\end{array}$ & $\begin{array}{c}2.56 \\
(1.52- \\
4.04) \\
\end{array}$ & $\begin{array}{l}18.64 \\
(14.66- \\
23.36) \\
\end{array}$ & $\begin{array}{c}2.14 \\
(1.24- \\
3.42) \\
\end{array}$ & $\begin{array}{c}16.56 \\
(12.91- \\
20.92) \\
\end{array}$ & $\begin{array}{l}1.80 \\
(1.01- \\
2.98) \\
\end{array}$ & $\begin{array}{l}11.93 \\
(9.03- \\
15.45) \\
\end{array}$ & $\begin{array}{c}1.26 \\
(0.60- \\
2.32) \\
\end{array}$ & $\begin{array}{c}19.14 \\
(15.39- \\
23.53) \\
\end{array}$ & $\begin{array}{c}0.96 \\
(0.46- \\
1.77) \\
\end{array}$ & $\begin{array}{l}11.61 \\
(8.53- \\
15.44) \\
\end{array}$ & $\begin{array}{c}2.49 \\
(1.48- \\
3.94) \\
\end{array}$ \\
\hline
\end{tabular}

a2016 U.S. dollars.

bEmergency department visits included visits that resulted in a hospitalization. These visits were counted as an emergency department visit and a hospitalization. $A E D=$ antiepileptic drug; $C B Z=$ carbamazepine; $C I=$ confidence interval $L C M=$ lacosamide; $L E V=$ levetiracetam; $L T G=$ lamotrigine; $M=$ maintenance; $P H T=$ phenytoin; $T=$ titration; $T P M=$ topiramate; $V P A=$ valproate; $U S D=U . S$. dollars. 